\title{
Biosensing studies on CuO-MgO nanocomposite for glucose detection
}

Najam US Sahar Riyaz ${ }^{1,2}$, Karthik Kannan', Vinotha Krishnasamy', John-John Cabibihan ${ }^{3}$, Abdulaziz Khalid AlAli $^{4}$, Rayaz A. Malik ${ }^{5}$, Kishor Kumar Sadasivuni ${ }^{*}$

${ }^{1}$ Centre for Advanced Materials, Qatar University, P.O. Box 2713, Doha, Qatar

${ }^{2}$ Chemistry and Earth Sciences, Qatar University, P.O. Box 2713, Doha, Qatar

${ }^{4}$ Department of Computer Engineering, Qatar University, P.O. Box 2713, Doha, Qatar. ${ }^{5}$ Weill Cornell Medicine, P.O. Box 2713, Doha, Qatar.

*kishorkumars@qu.edu.qa

\section{ABSTRACT}

Approximately 3 million people around the world suffer from diabetes. One of the basic indications of an individual suffering from diabetes can be observed in the form of peaked levels of glucose in the blood. Thus, it is imperative for a non-invasive mechanism to be derived through which glucose levels in the blood can be detected throughout a regular time frame. The aim of this project focuses on synthesis of a nanocomposite which can be used to detect glucose levels in the blood in a non-invasive manner. The selected nanocomposite was made from a mixture of $\mathrm{CuO}$ and $\mathrm{MgO}$. Once synthesized, it was subjected to a series of tests and scans. The results demonstrated effective and efficient glucose detection activity of the $\mathrm{CuO}-\mathrm{MgO}$ nanocomposite.

\section{INTRODUCTION}

The current methods for glucose detection are mainly invasive techniques which require obtaining a sample of blood from the patient.

$>$ Nanocomposites can be effectively used for non-invasive detection of glucose in the blood for early detection and constant monitoring in any individual suffering from diabetes.

\section{METHODOLOGY}
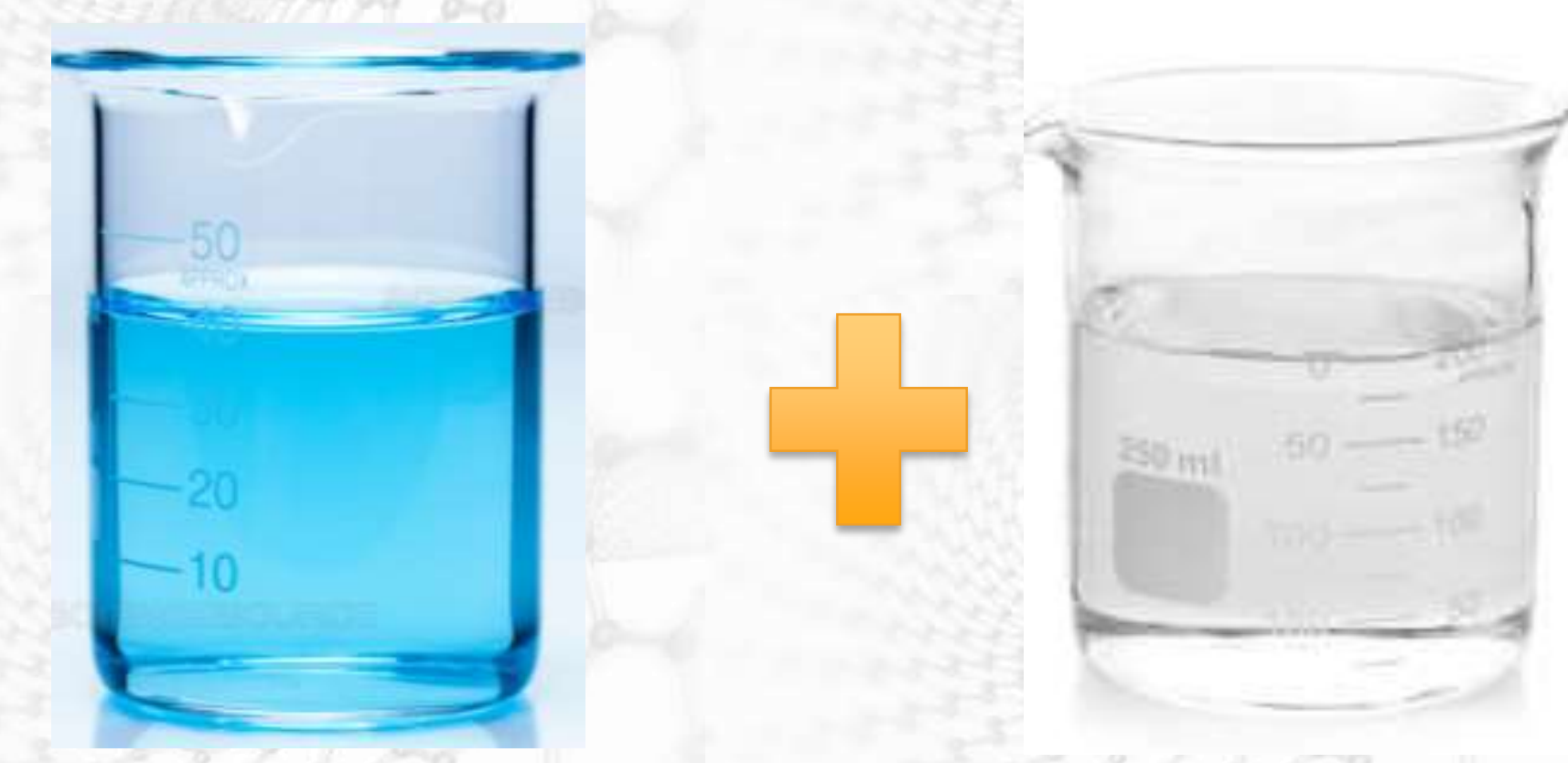

$0.5 \mathrm{M} \mathrm{Cu}\left(\mathrm{NO}_{3}\right)_{2}+\mathrm{DD} \mathrm{H}_{2} \mathrm{O} \quad 0.5 \mathrm{M} \mathrm{Mg}\left(\mathrm{NO}_{3}\right)_{2}+\mathrm{DD} \mathrm{H}_{2} \mathrm{O}$

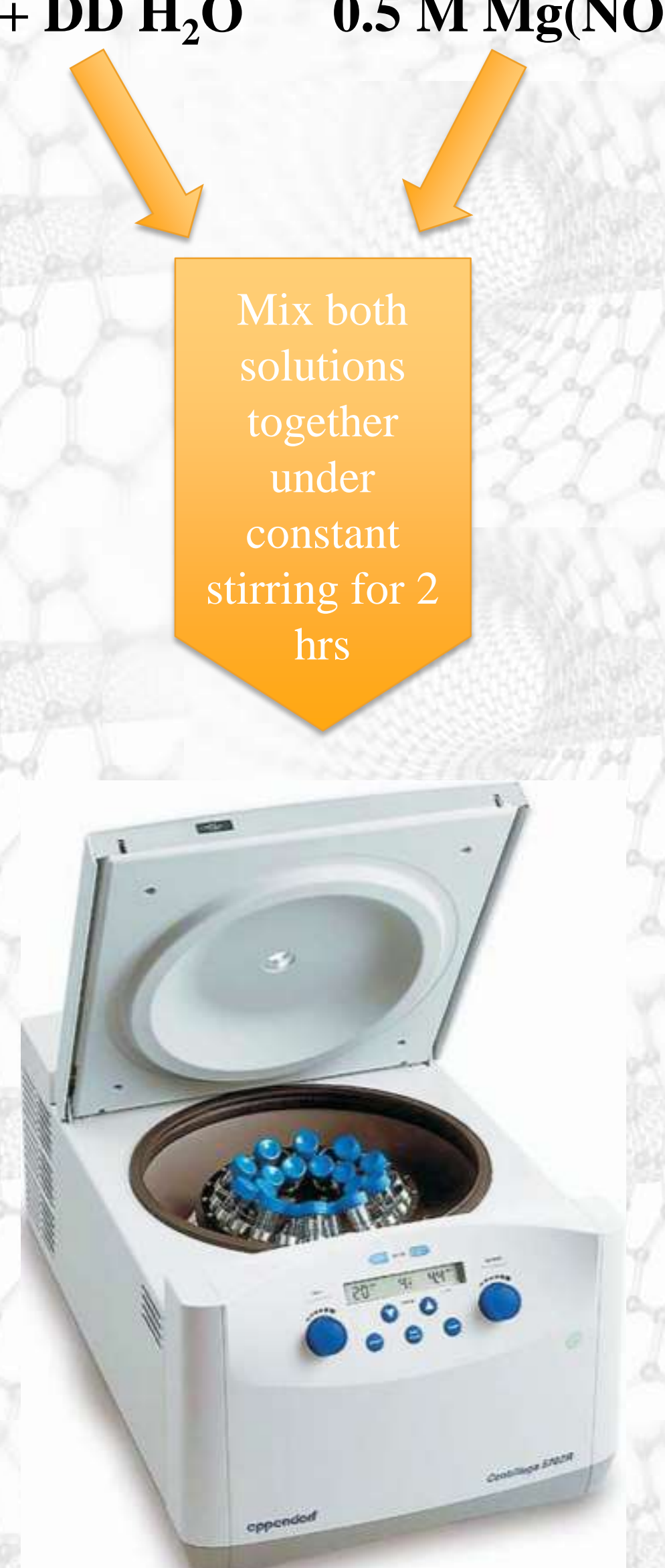

The resulting solution was centrifuged at 3000rpm

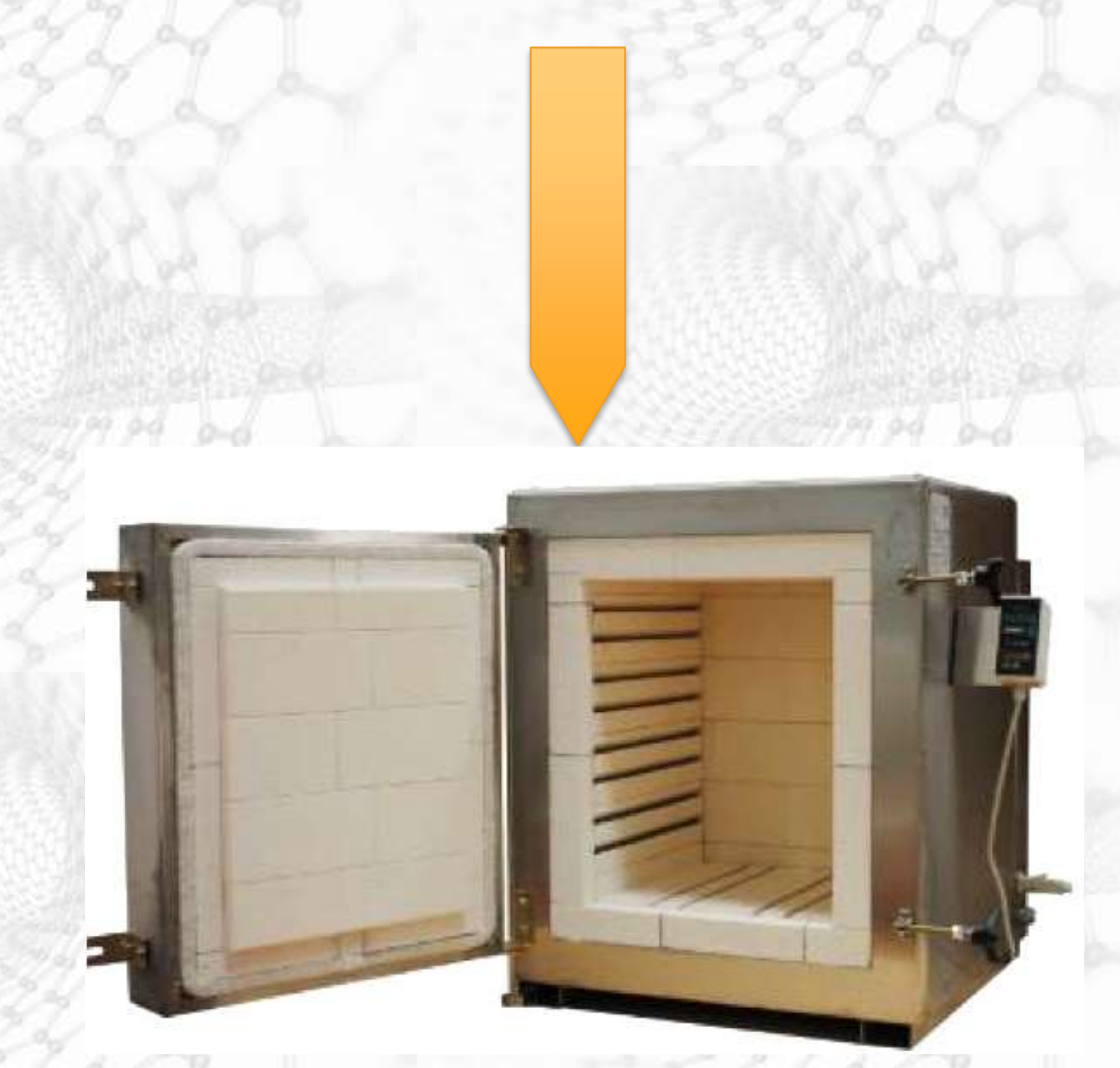

Calcinated at $500{ }^{\circ} \mathrm{C}$ for $4 \mathrm{hrs}$

\section{RESULTS \& DISCUSSION}

Structural Studies

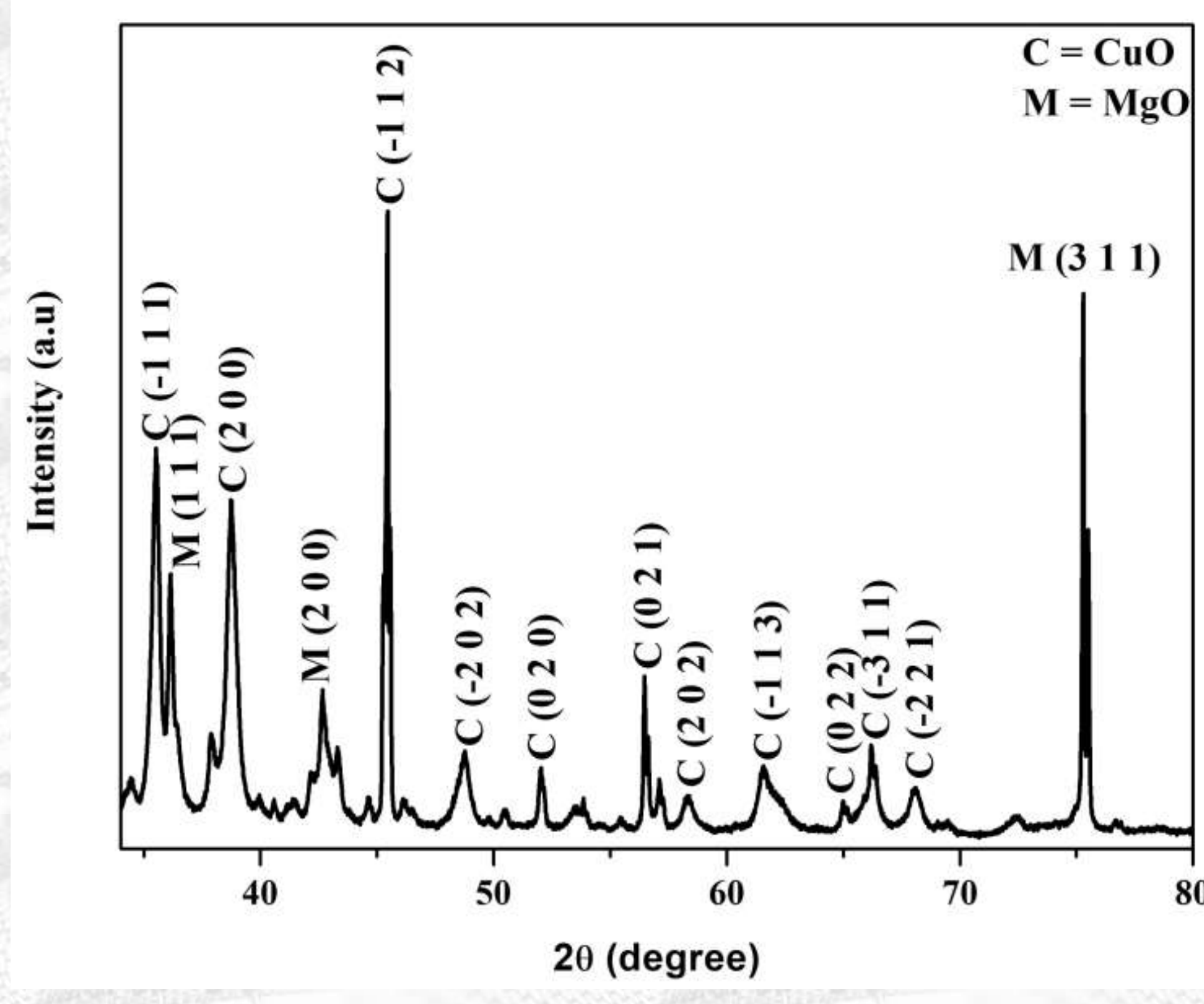

X-Ray Diffraction Results of the synthesized nanocomposite

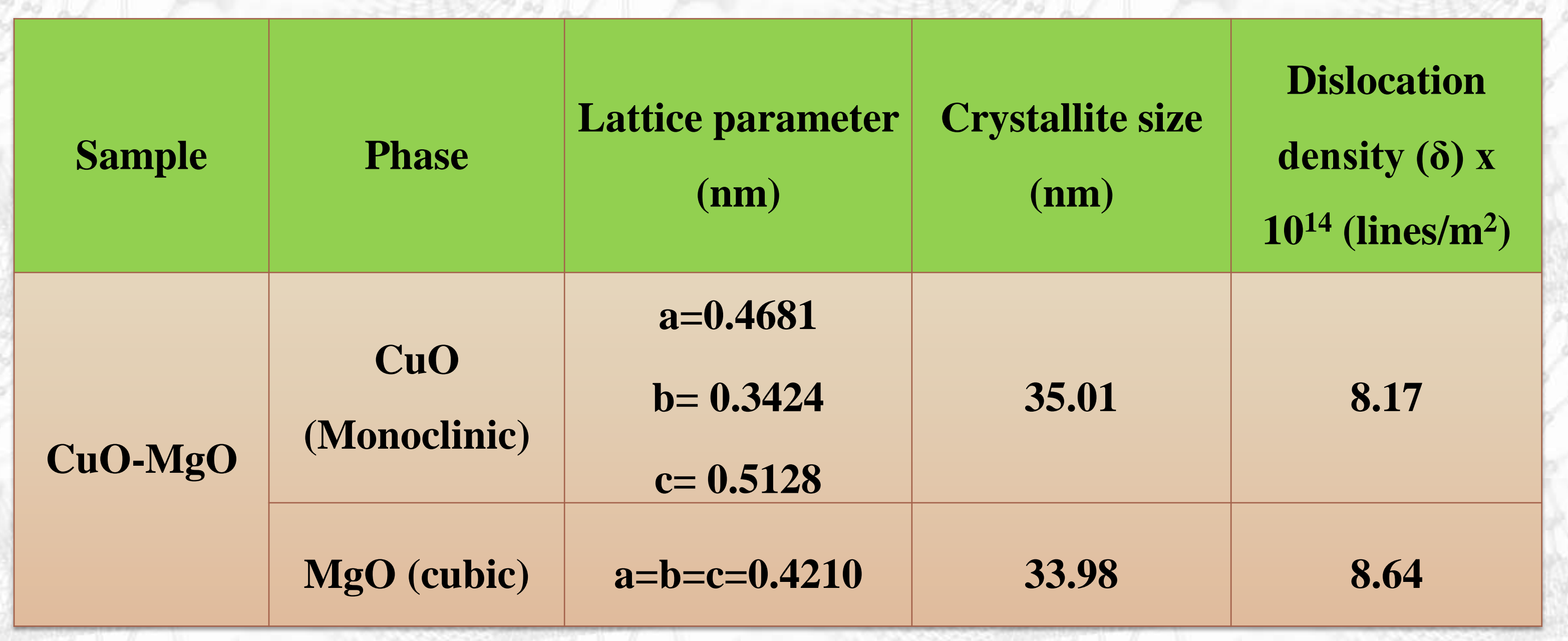

Lattice parameters obtained from $\mathrm{CuO}-\mathrm{MgO}$ nanocomposite
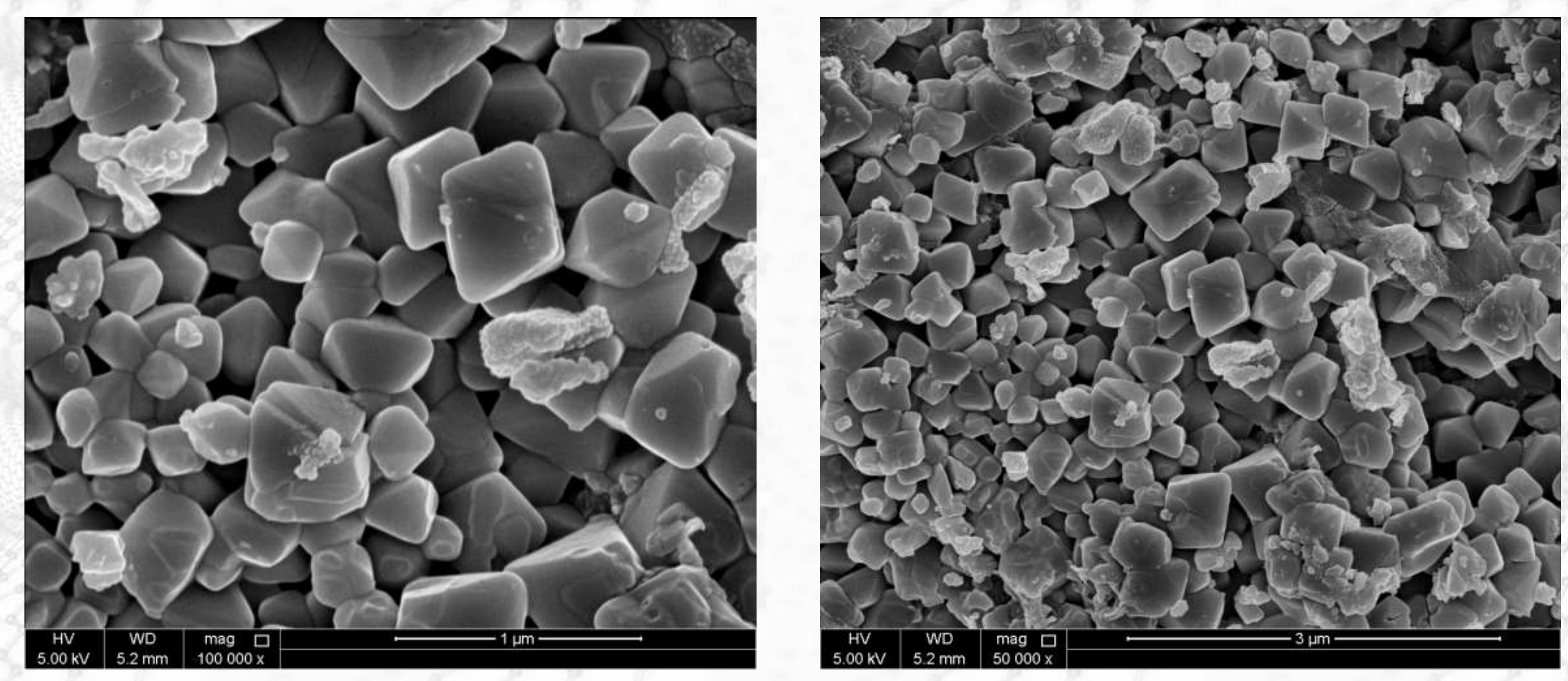

SEM - CuO-MgO nanocomposite - Kidney stone monoclinic crystals

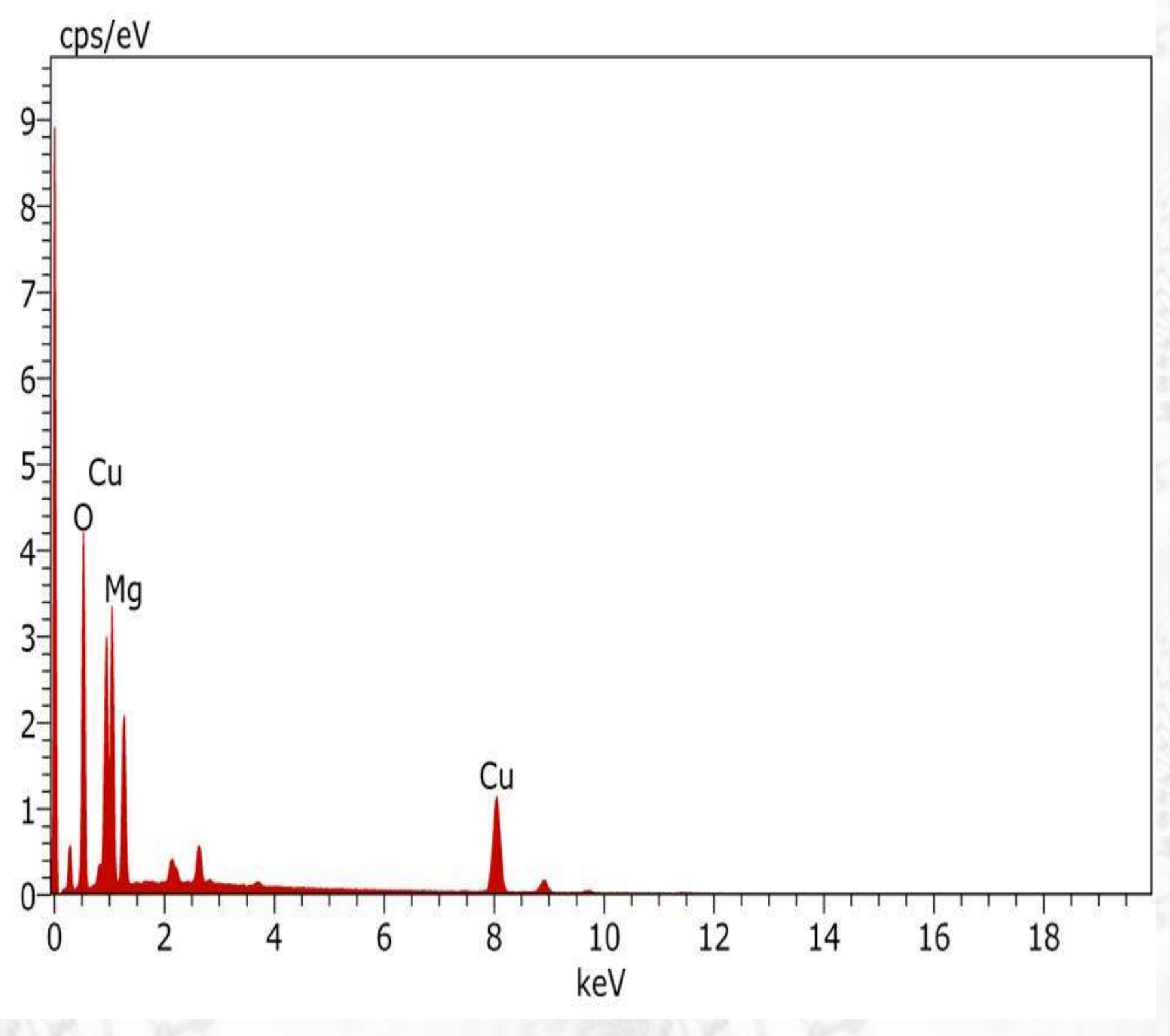

EDAX results showing only the presence of $\mathrm{Cu}, \mathrm{Mg}$ and $\mathrm{O}$ in the nanocomposites thus proving the purity of the nanocomposites

\begin{tabular}{|c|c|c|}
\hline Element & Wt $(\%)$ & At $(\%)$ \\
\hline $\mathrm{Cu}$ & 22.27 & 74.42 \\
\hline $\mathrm{Mg}$ & 21.07 & 18.21 \\
\hline $\mathrm{O}$ & 56.66 & 7.37 \\
\hline
\end{tabular}

\section{Raman Studies}

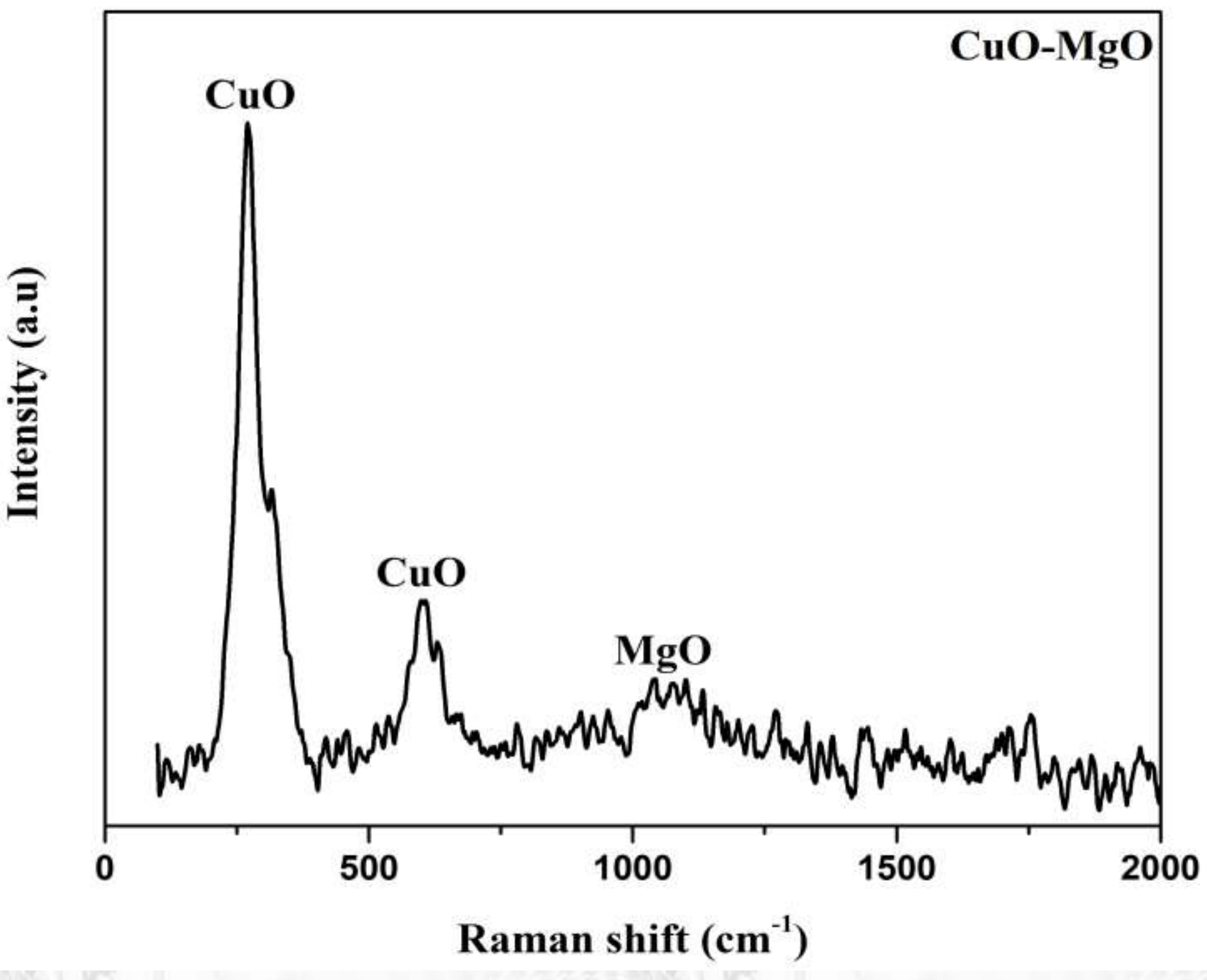
presence of $\mathrm{CuO}$ and $\mathrm{MgO}$ at the corresponding intensity values
Tests for Biosensing Properties

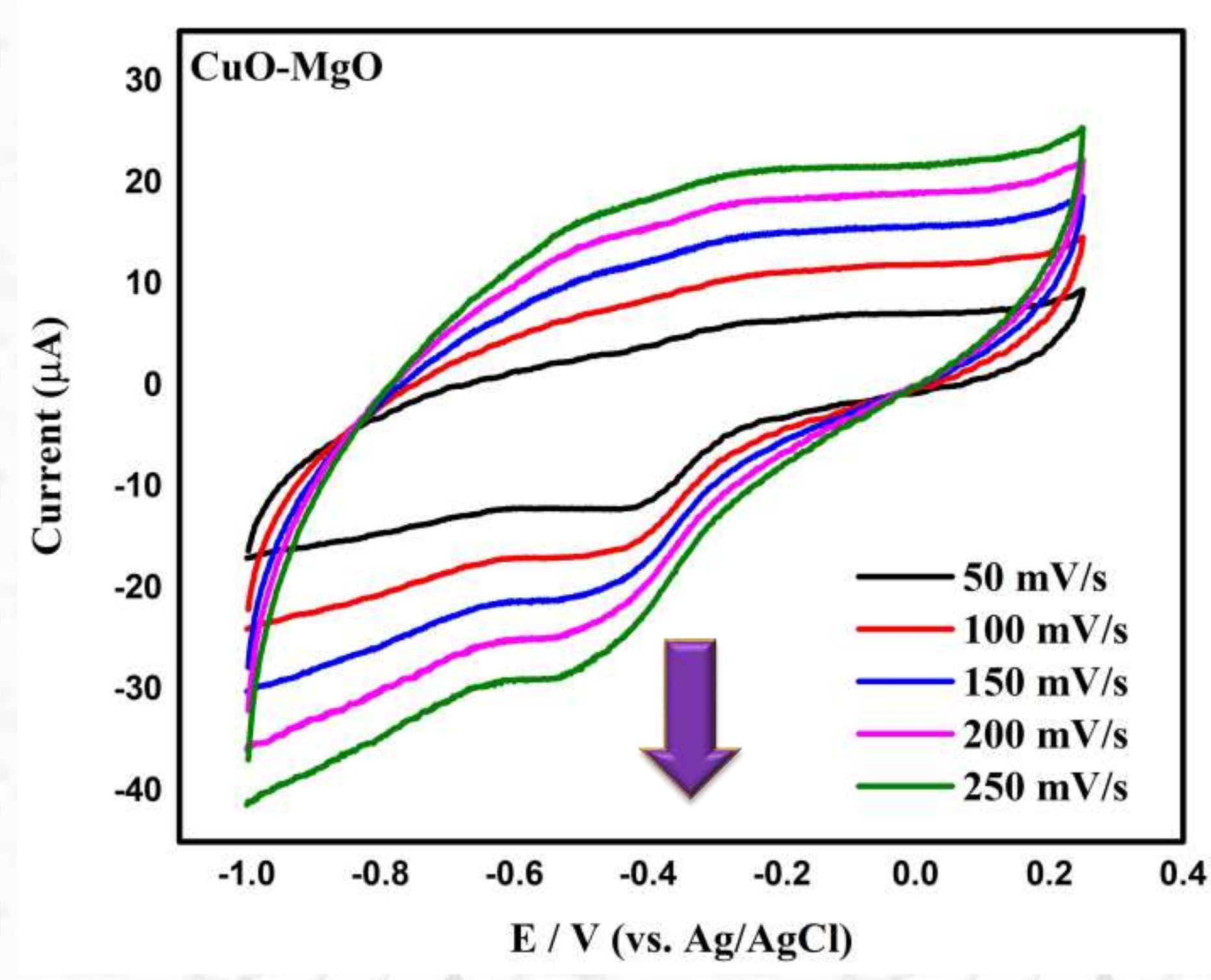

$\mathrm{CV}$ with different scan rates

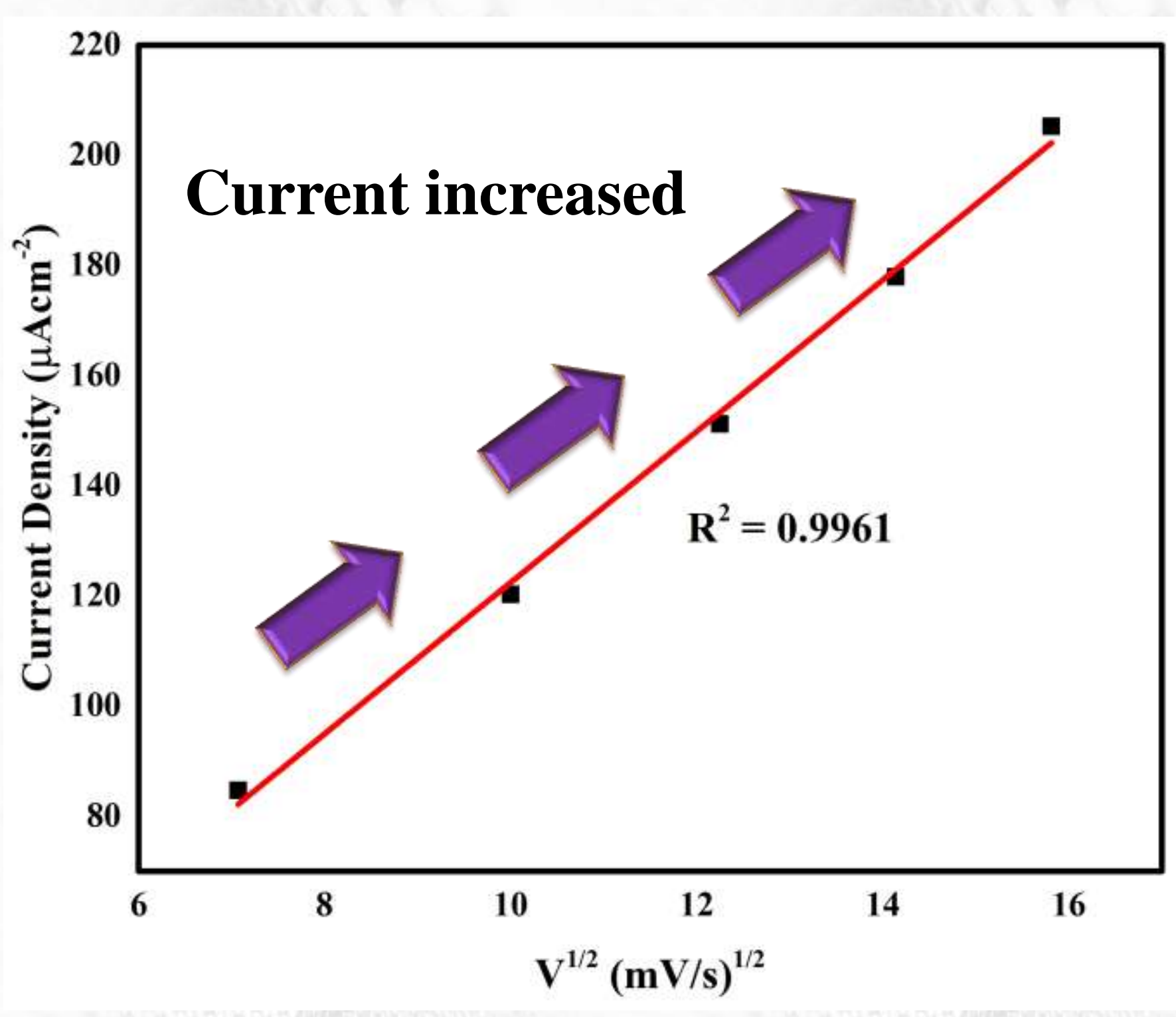

Current density measured at different scan rates

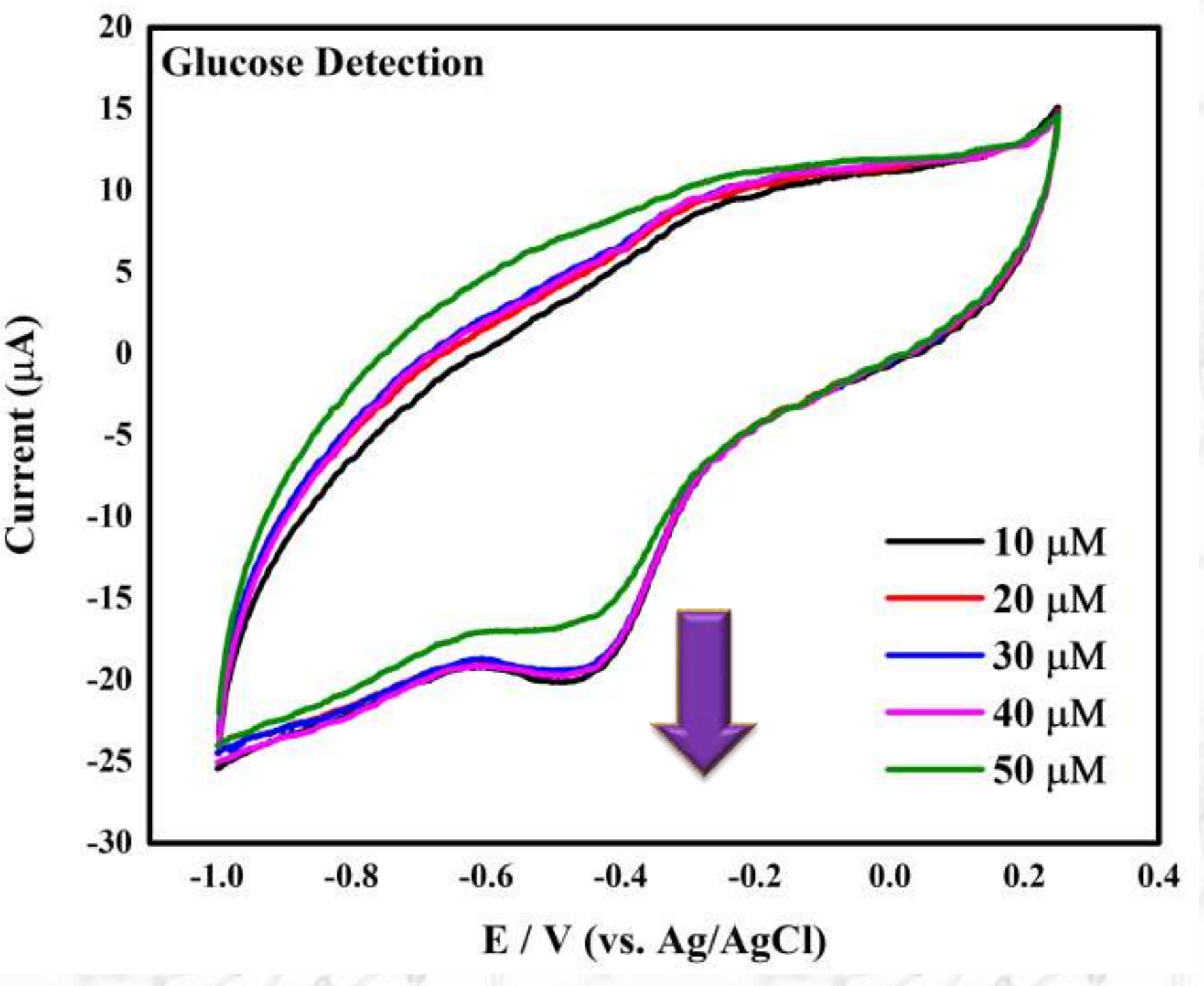

Scan for glucose detection

\section{BENEFITS TO QATAR}

Given the increasing population with health issues, in particular diabetes, a non-invasive method to detect the levels of glucose in blood is imperative. Using such a nanocomposite is the first step towards achieving this method of effective and efficient detection of glucose levels in the blood so that any abnormal patterns or fluctuations may be detected as well as for the purposes of early detection of diabetes. Furthermore, it can also be used to detect/monitor if an individual with a previous family history of diabetes has high levels of glucose in their blood so that they can prevent diabetes or at the very least, delay it.

\section{CONCLUSION}

XRD: $\mathrm{CuO}$ (monoclinic), $\mathrm{MgO}$ (cubic) structure SEM with EDAX: Kidney stone monoclinic crystals Raman: 270 (CuO), $600(\mathrm{CuO}), 1071 \mathrm{~cm}^{-1}(\mathrm{MgO})$

Biosensors: The modified electrode shows a sensitivity $0.48 \mu \mathrm{A} \mathrm{cm}^{-2} \mu \mathrm{M}^{-1}$

Glucose sensor development is important for diabetic Patients.

\section{REFERENCES}

1. Velmurugan, A., Jeevandass, S., Simon, F. J., \& Bhojan, L. (2015). Sweat Based Glucose Analysis. International Journal for Research in Applied Science \& Engineering Technology, 3(3), 550-555.

2. Karthik Kannan, S. Dhanuskodi, S. Prabukumar, S. Sivaramakrishnan, Optik 204 (2020) 164221.

\section{ACKNOWLEDGEMENTS}

This work was supported by the NPRP grant \# NPRP11S-0110-180247 from the Qatar National Research Fund (a member of Qatar Foundation). The statements made herein are solely the responsibility of the authors Thanks are also to Central Laboratory Unit, Qatar University, Qatar. 